\title{
Identification of hypo- and hypermethylated genes related to atherosclerosis by a genome-wide analysis of DNA methylation
}

\author{
YOSHIJI YAMADA ${ }^{1}$, TAMOTSU NISHIDA ${ }^{1}$, HIDEKI HORIBE ${ }^{2}$, \\ MITSUTOSHI OGURI $^{3}$, KIMIHIKO KATO ${ }^{1,4}$ and MOTOJI SAWABE ${ }^{5}$ \\ ${ }^{1}$ Department of Human Functional Genomics, Life Science Research Center, Mie University, Tsu, Mie; \\ ${ }^{2}$ Department of Cardiovascular Medicine, Gifu Prefectural Tajimi Hospital, Tajimi, Gifu; ${ }^{3}$ Department of Cardiology, \\ Japanese Red Cross Nagoya First Hospital, ${ }^{4}$ Department of Internal Medicine, Meitoh Hospital, Nagoya, Aichi; \\ ${ }^{5}$ Section of Molecular Pathology, Graduate School of Health Care Sciences, \\ Tokyo Medical and Dental University, Tokyo, Japan
}

Received November 30, 2013; Accepted March 4, 2014

DOI: $10.3892 / \mathrm{ijmm} .2014 .1692$

\begin{abstract}
Epigenetic modification, particularly changes in DNA methylation at gene promoters, is implicated in the pathogenesis of atherosclerosis. However, the analysis of DNA methylation in atherosclerosis has been limited to a few selected candidate genes. In this study, we therefore performed a genome-wide analysis of DNA methylation in the atherosclerotic human aorta. A total of 48 post-mortem human aortic intima specimens were examined. To avoid the effects of interindividual variation, we performed intraindividual paired comparisons between atheromatous plaque lesions and corresponding plaque-free tissue for 24 subjects. Bisulfitemodified genomic DNA was analyzed for DNA methylation with a specific microarray (Illumina HumanMethylation450 BeadChip). We compensated for multiple comparisons by applying Bonferroni's correction for statistical significance of association. DNA methylation was significantly $\left(\mathrm{P}<1.03 \times 10^{-7}\right)$ reduced at $15 \mathrm{CpG}$ sites in 14 genes and increased at $30 \mathrm{CpG}$ sites in 22 genes in atheromatous plaque compared with plaquefree intima. Three of the hypomethylated genes [Drosophila headcase $(H E C A)$, early B-cell factor $1(E B F 1)$ and nucleotidebinding oligomerization domain containing 2 (NOD2)] and three of the hypermethylated genes [human mitogen-activated protein kinase kinase kinase kinase 4 (MAP4K4), zinc finger E-box binding homeobox 1 (ZEBI) and $F Y N]$ were previously been implicated in atherosclerosis. The overexpression of $H E C A, E B F 1$ or $N O D 2$ or the suppression of $M A P 4 K 4$,
\end{abstract}

Correspondence to: Professor Yoshiji Yamada, Department of Human Functional Genomics, Life Science Research Center, Mie University, 1577 Kurima-machiya, Tsu, Mie 514-8507, Japan E-mail: yamada@gene.mie-u.ac.jp

Key words: atherosclerosis, cardiovascular disease, DNA methylation, epigenetics, epigenome-wide association study
ZEB1 or FYN expression in cultured HEK293 cells resulted in significant $\left(\mathrm{P}<4.80 \times 10^{-7}\right)$ changes in the expression of atherosclerosis-related genes, as determined with an expression microarray (Illumina HumanHT-12 v4 Expression BeadChip). Our findings suggested that $H E C A, E B F 1$ and NOD2 were significantly hypomethylated, whereas $M A P 4 K 4, Z E B 1$ and $F Y N$ were hypermethylated, in atheromatous plaque lesions compared with plaque-free intima. Epigenetic mechanisms may thus contribute to the pathogenesis of atherosclerosis.

\section{Introduction}

Epigenetic modification, particularly changes in DNA methylation at gene promoters, has been implicated in the pathogenesis of various complex diseases, including atherosclerotic cardiovascular disease (1-4). Given that cellular patterns of DNA methylation are affected by environmental and dietary factors, as well as by age, gender and genetic variants $(1,2)$, knowledge of the DNA methylation pattern of atherosclerotic plaque lesions may provide insight into the molecular mechanisms and interindividual outcome variability of atherosclerotic diseases. Although DNA methylation in various genes has been shown to be related to atherosclerosis or cardiovascular disease (4-6), the pattern of DNA methylation in the atherosclerotic human aorta has not been fully determined at the genome-wide level. In this study, we therefore performed a genome-wide analysis of DNA methylation in the atherosclerotic human aorta in order to clarify epigenetic mechanisms underlying the development of atherosclerosis.

\section{Materials and methods}

Study samples. A total of 48 post-mortem specimens of the human aortic intima were examined. To avoid the effects of interindividual variation, we performed intraindividual paired comparisons of DNA methylation between atheromatous plaque lesions and corresponding plaque-free tissue for 24 subjects. The tissue samples were frozen at $-80^{\circ} \mathrm{C}$ immediately after dissection. The study protocol was approved by 
the Committees on the Ethics of Human Research of Mie University Graduate School of Medicine, Tokyo Metropolitan Institute of Gerontology, Japanese Red Cross Nagoya First Hospital (Nagoya, Japan), and Gifu Prefectural Tajimi Hospital (Tajimi, Japan). Written informed consent was obtained from families of the deceased subjects.

Immunohistochemical analysis of atheromatous plaque and plaque-free intima. Specimens of atheromatous plaque lesions and plaque-free intima were subjected to immunohistochemical analysis. Formalin-fixed and paraffin-embedded sections were depleted of paraffin, hydrated, immersed in $0.01 \mathrm{~mol} / 1$ citrate buffer ( $\mathrm{pH} 6.0$ ), and heated for $10 \mathrm{~min}$ in a pressure cooker. Staining was performed with the use of a ChemMate Envision/HRP kit (Dako, Glostrup, Denmark). Mouse monoclonal antibodies to $\alpha$-smooth muscle actin ( $\alpha$-SMA) (clone 1A4, M0851; Dako), to CD68 (clone PG-M1, N1576; Dako) and to CD45 (clone 2B11 + PD7/26, 722071; Nichirei Bioscience, Tokyo, Japan) were applied according to the manufacturer's instructions. Proteinase K pre-treatment was used for CD68 and CD45.

Genome-wide analysis of DNA methylation. Genomic DNA was extracted from finely minced tissue specimens with phenol-chloroform and was then precipitated with ethanol. Bisulfite-modified genomic DNA was analyzed for DNA methylation with a DNA methylation-specific microarray that includes $485,553 \mathrm{CpG}$ sites distributed throughout the entire genome (HumanMethylation450 BeadChip; Illumina, San Diego, CA, USA). Bisulfite conversion was performed with an EZ DNA Methylation kit (Zymo Research, Irvine, CA, USA). We measured methylation at $\mathrm{CpG}$ sites in genomic DNA isolated from atheromatous plaque or plaque-free intima with a GenomeStudio Methylation Module (Illumina). Call rate values for the 48 samples were $\geq 99.3 \%$, with a mean of $99.9 \%$. The DNA methylation level at each $\mathrm{CpG}$ site was calculated as the $\beta$ value, where $\beta=$ intensity of the methylated allele/ (intensity of the methylated allele + intensity of the unmethylated allele +100$)$, as previously described $(7,8)$.

Transfection and immunoblot analysis. Human embryonic kidney (HEK) 293 cells were cultured under $5 \% \mathrm{CO}_{2}$ at $37^{\circ} \mathrm{C}$ in Dulbecco's modified Eagle's medium supplemented with $10 \%$ fetal bovine serum, penicillin $(100 \mathrm{U} / \mathrm{ml})$ and streptomycin $(100 \mu \mathrm{g} / \mathrm{ml})$. For examination of the effects of gene overexpression, HEK 293 cells were transfected for $48 \mathrm{~h}$ with the expression vector, pCMV6-Entry (encoding $\mathrm{COOH}$-terminal Myc and DDK epitope tags; OriGene, Rockville, MD, USA), containing human Drosophila headcase (HECA) homolog, early B-cell factor 1 (EBF1), or nucleotide-binding oligomerization domain containing 2 (NOD2) cDNA (or with the empty vector alone) with the use of polyethylenimine, as previously described (9). The transfected cells were solubilized with $2 \mathrm{X}$ sodium dodecyl sulfate (SDS) sample buffer and subjected to immunoblot analysis with antibodies to human HECA (ab98993), EBF1 (ab126135), or NOD2 (ab31488) (all from Abcam, Cambridge, UK) at a dilution of 1:1,000, 1:1,000 or 1:500, respectively, or with mouse monoclonal antibodies to the DDK epitope (OriGene) at a dilution of 1:6,000. For the examination of the effects of attenuation of gene expres- sion, HEK293 cells were transfected for $72 \mathrm{~h}$ with the vector, pGFP-V-RS encoding short hairpin RNA (shRNA) for human mitogen-activated protein kinase kinase kinase kinase 4 (MAP4K4), zinc finger E-box binding homeobox 1 (ZEBI) or $F Y N$ or a scrambled shRNA (OriGene), with the use of polyethylenimine, as previously described (9). The cells were then lysed and subjected to immunoblot analysis with antibodies to human MAP4K4 (ab134092), ZEB1 (ab155249), or FYN (ab119855) (all from Abcam) at a dilution of 1:1,000, 1:1,000 or 1:500, respectively. Immune complexes were detected with enhanced chemiluminescence reagents (GE Healthcare Bio-Science, Piscataway, NJ, USA).

Genome-wide gene expression analysis. Total RNA was isolated from the transfected cells with the use of a NucleoSpin RNA II kit (Macherey-Nagel, Düren, Germany). A total of 72 RNA samples was analyzed for RNA concentration and RNA integrity (RIN) with a 2100 Bioanalyzer and an RNA 6000 Nano kit (Agilent Technologies, Santa Clara, CA, USA). The RIN values for the 72 samples were $\geq 9.4$, with a mean of 9.93. The RNA samples were then subjected to genome-wide analysis of gene expression with a microarray that targets 34,694 transcripts corresponding to well-characterized genes, gene candidates, or splice variants distributed throughout the entire genome (HumanHT-12 v4 Expression BeadChip; Illumina). In brief, total RNA (500 ng) was amplified as cRNA and biotinylated with the use of Epicentre TargetAmp Nano-g Biotin-aRNA Labeling for the Illumina System (Epicentre, Madison, WI, USA). The concentration and quality of the biotinylated cRNA were assessed with the 2100 Bioanalyzer. Subsequent steps included hybridization of each sample to the HumanHT-12 v4 Expression BeadChip, washing, blocking and streptavidin-Cy3 staining. A GenomeStudio Gene Expression Module (Illumina) was used to generate signal intensity values from the scans and to perform the initial quality controls. Values for noise-to-signal ratio (P95/P05 ratio) were between 12.8 and 23.8 for all samples (mean, 17.4), which is within the acceptable range of $\geq 10$.

Statistical analysis. DNA methylation ( $\beta$ values) or gene expression data were compared between two groups with the unpaired Student's t-test or among three or more groups by one-way analysis of variance, respectively. To compensate for multiple comparisons, we applied Bonferroni's correction for statistical significance of association. The significance level was thus $\mathrm{P}<1.03 \times 10^{-7}(0.05 / 485,553)$ for the genome-wide analysis of DNA methylation (Table I) or $\mathrm{P}<4.80 \times 10^{-7}[0.05 /(34,694 \times 3)]$ for the genome-wide analysis of gene expression (Tables II and III). Statistical tests were performed with JMP Genomics version 6.0 software (SAS Institute, Inc., Cary, NC, USA).

\section{Results}

Immunohistochemical analysis of atheromatous plaque and plaque-free intima. We performed immunohistochemical staining for SMA, CD68 and CD45 (markers for smooth muscle cells, macrophages and lymphocytes, respectively) in specimens of the human aorta containing atheromatous plaque lesions or plaque-free intima. Atheromatous plaque lesions appeared positive for SMA and CD68, but negative for CD45. 
Table I. Identification by genome-wide analysis of $\mathrm{CpG}$ sites whose methylation status is significantly related to atherosclerosis.

\begin{tabular}{|c|c|c|c|c|c|c|c|c|}
\hline Chromosome & Gene & $\mathrm{CpG}$ & $\begin{array}{l}\text { Relation } \\
\text { to } \mathrm{CpG}\end{array}$ & $\begin{array}{l}\text { Methylation } \\
\text { site }\end{array}$ & $\begin{array}{l}\text { Mean } \beta \text { value } \\
\text { (plaque) }\end{array}$ & $\begin{array}{c}\text { Mean } \beta \text { value } \\
\text { (plaque-free) }\end{array}$ & $\begin{array}{c}\beta \text { ratio } \\
\text { (plaque/plaque-free) }\end{array}$ & P-value \\
\hline 17 & SEPT9 & $\operatorname{cg} 14885762$ & N Shore & TSS200 & 0.6283 & 0.5176 & 1.21 & $2.4 \times 10^{-9}$ \\
\hline 15 & KIAA1199 & cg02240539 & & 5'UTR & 0.7175 & 0.5579 & 1.29 & $6.3 \times 10^{-9}$ \\
\hline 6 & & $\operatorname{cg} 04304054$ & & & 0.6749 & 0.5212 & 1.29 & $1.7 \times 10^{-8}$ \\
\hline 6 & $A R I D I B$ & $\operatorname{cg} 17164954$ & S Shelf & Body & 0.2551 & 0.3716 & 0.69 & $2.2 \times 10^{-8}$ \\
\hline 1 & $S L C 2 A 1$ & $\operatorname{cg} 16738646$ & & Body & 0.2392 & 0.3977 & 0.60 & $2.2 \times 10^{-8}$ \\
\hline 2 & $M A P 4 K 4$ & $\operatorname{cg} 05113410$ & & Body & 0.6508 & 0.5307 & 1.23 & $2.4 \times 10^{-8}$ \\
\hline 11 & ST5 & $\operatorname{cg} 14521421$ & & 5'UTR & 0.7343 & 0.6232 & 1.18 & $2.4 \times 10^{-8}$ \\
\hline 6 & $H E C A$ & $\operatorname{cg} 08943714$ & & Body & 0.2662 & 0.4037 & 0.66 & $2.8 \times 10^{-8}$ \\
\hline 9 & SMC5 & $\operatorname{cg} 14477581$ & & Body & 0.5906 & 0.4710 & 1.25 & $3.2 \times 10^{-8}$ \\
\hline 1 & MIR34A & cg00909706 & & TSS200 & 0.7355 & 0.6047 & 1.22 & $3.2 \times 10^{-8}$ \\
\hline 10 & & $\operatorname{cg} 12714759$ & N Shelf & & 0.1737 & 0.2626 & 0.66 & $3.6 \times 10^{-8}$ \\
\hline 4 & & $\operatorname{cg} 24592513$ & & & 0.2093 & 0.3112 & 0.67 & $3.8 \times 10^{-8}$ \\
\hline 2 & & $\operatorname{cg} 00716848$ & & & 0.8584 & 0.7257 & 1.18 & $3.9 \times 10^{-8}$ \\
\hline 5 & AP3S1 & $\operatorname{cg} 24770230$ & & Body & 0.7915 & 0.6997 & 1.13 & $4.0 \times 10^{-8}$ \\
\hline 10 & $B I C C 1$ & $\operatorname{cg} 08466030$ & & Body & 0.7054 & 0.6176 & 1.14 & $4.1 \times 10^{-8}$ \\
\hline 1 & & $\operatorname{cg} 22046201$ & & & 0.3712 & 0.5305 & 0.70 & $4.3 \times 10^{-8}$ \\
\hline 17 & SEPT9 & $\operatorname{cg} 20772590$ & N Shore & TSS200 & 0.6167 & 0.5181 & 1.19 & $4.4 \times 10^{-8}$ \\
\hline 10 & $Z E B 1$ & $\operatorname{cg} 18516609$ & & Body & 0.7518 & 0.6440 & 1.17 & $4.5 \times 10^{-8}$ \\
\hline 1 & CAMTA1 & $\operatorname{cg} 24634746$ & & Body & 0.7967 & 0.6622 & 1.20 & $4.5 \times 10^{-8}$ \\
\hline 17 & MIR1180 & cg26619894 & S Shore & TSS 1500 & 0.7982 & 0.7177 & 1.11 & $4.7 \times 10^{-8}$ \\
\hline 2 & $G L S$ & $\operatorname{cg} 03962451$ & & Body & 0.3923 & 0.5382 & 0.73 & $4.7 \times 10^{-8}$ \\
\hline 5 & $E B F 1$ & $\operatorname{cg} 21211213$ & & Body & 0.1954 & 0.2643 & 0.74 & $4.7 \times 10^{-8}$ \\
\hline 19 & PTPRS & $\operatorname{cg} 08462941$ & N Shore & 5'UTR & 0.6487 & 0.5613 & 1.16 & $4.8 \times 10^{-8}$ \\
\hline 17 & $A B R$ & $\operatorname{cg} 16374343$ & S Shore & Body & 0.2230 & 0.3544 & 0.63 & $4.9 \times 10^{-8}$ \\
\hline 15 & LOC145845 & $\operatorname{cg} 12288941$ & S Shelf & Body & 0.1361 & 0.1978 & 0.69 & $5.1 \times 10^{-8}$ \\
\hline 4 & SH3D19 & $\operatorname{cg} 12556802$ & & 5'UTR & 0.7845 & 0.6322 & 1.24 & $5.6 \times 10^{-8}$ \\
\hline 8 & $D L C 1$ & $\operatorname{cg} 22045977$ & & Body & 0.8009 & 0.6945 & 1.15 & $5.9 \times 10^{-8}$ \\
\hline 17 & & $\operatorname{cg} 10586883$ & N Shelf & & 0.6865 & 0.5469 & 1.26 & $5.9 \times 10^{-8}$ \\
\hline 12 & PPMIH & cg06208382 & & Body & 0.3753 & 0.5020 & 0.75 & $6.2 \times 10^{-8}$ \\
\hline 6 & $F Y N$ & $\operatorname{cg} 08114265$ & & 5'UTR & 0.7132 & 0.6451 & 1.11 & $6.4 \times 10^{-8}$ \\
\hline 10 & BTBD16 & $\operatorname{cg} 01077100$ & & Body & 0.2701 & 0.3846 & 0.70 & $6.6 \times 10^{-8}$ \\
\hline 16 & NOD2 & $\operatorname{cg} 18177814$ & & TSS200 & 0.2629 & 0.3309 & 0.79 & $6.8 \times 10^{-8}$ \\
\hline 7 & $R A D I L$ & cg20556639 & N Shelf & 5'UTR & 0.7947 & 0.6981 & 1.14 & $7.0 \times 10^{-8}$ \\
\hline 22 & & cg09349128 & N Shore & & 0.2440 & 0.3571 & 0.68 & $7.5 \times 10^{-8}$ \\
\hline 14 & & cg20323874 & & & 0.6645 & 0.5561 & 1.20 & $8.4 \times 10^{-8}$ \\
\hline 7 & $R N F 216$ & $\operatorname{cg} 26724841$ & N Shelf & 5'UTR & 0.6620 & 0.5734 & 1.15 & $8.7 \times 10^{-8}$ \\
\hline 16 & & cg02196592 & & & 0.5947 & 0.4921 & 1.21 & $8.9 \times 10^{-8}$ \\
\hline 15 & & $\operatorname{cg} 16906765$ & S Shelf & & 0.6233 & 0.5420 & 1.15 & $8.9 \times 10^{-8}$ \\
\hline 16 & & $\operatorname{cg} 27647755$ & & & 0.8287 & 0.7436 & 1.11 & $9.2 \times 10^{-8}$ \\
\hline 2 & & $\operatorname{cg} 01473038$ & N Shore & & 0.7275 & 0.6364 & 1.14 & $1.0 \times 10^{-7}$ \\
\hline 10 & $M I C U 1$ & cg09553839 & & 5'UTR & 0.4328 & 0.5278 & 0.82 & $1.0 \times 10^{-7}$ \\
\hline 7 & CPEDI & $\operatorname{cg} 18952945$ & & Body & 0.8271 & 0.7689 & 1.08 & $1.0 \times 10^{-7}$ \\
\hline 15 & $R A B 8 B$ & $\operatorname{cg} 09251291$ & S Shore & Body & 0.6936 & 0.5393 & 1.29 & $1.0 \times 10^{-7}$ \\
\hline 13 & ENOX1 & $\operatorname{cg} 24797276$ & & 5'UTR & 0.7984 & 0.7400 & 1.08 & $1.0 \times 10^{-7}$ \\
\hline 10 & COL13A1 & cg20740485 & & Body & 0.7139 & 0.6019 & 1.19 & $1.0 \times 10^{-7}$ \\
\hline
\end{tabular}

Genome-wide analysis of DNA methylation was performed for genomic DNA from atheromatous plaque and matched plaque-free intima specimens of the postmortem human aorta from 24 subjects. Hypomethylated or hypermethylated CpG sites significantly $\left(\mathrm{P}<1.03 \times 10^{-7}\right)$ related to atherosclerosis are listed. TSS200 $(1500)$, within 200 (1500) bp from the transcription start site; UTR, untranslated region.

Higher-magnification images revealed that infiltrated foamy macrophages were abundant, whereas lymphocytes were rare in atheromatous plaque. Plaque-free intima was positive for SMA, but negative for CD68 and CD45 (data not shown).
Genome-wide analysis of DNA methylation in atheromatous plaque and corresponding plaque-free intima. The extent of DNA methylation was significantly $\left(\mathrm{P}<1.03 \times 10^{-7}\right)$ reduced at $15 \mathrm{CpG}$ sites in 14 genes and significantly increased at $30 \mathrm{CpG}$ 
A

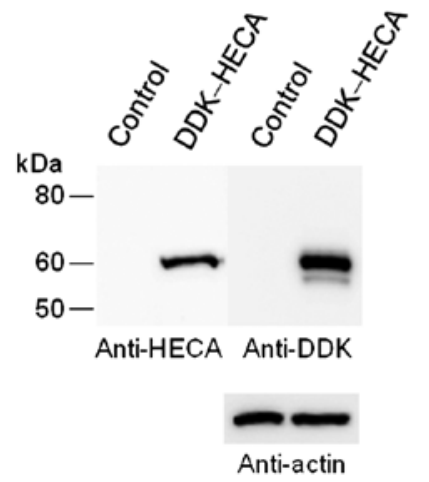

B

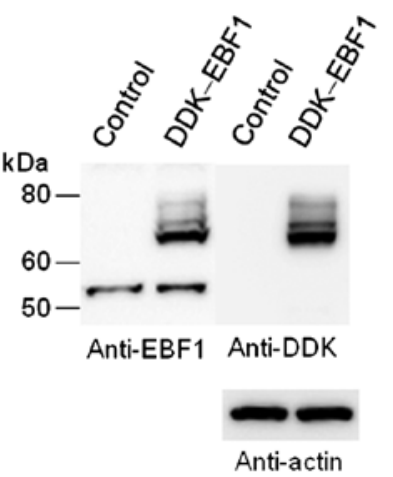

C

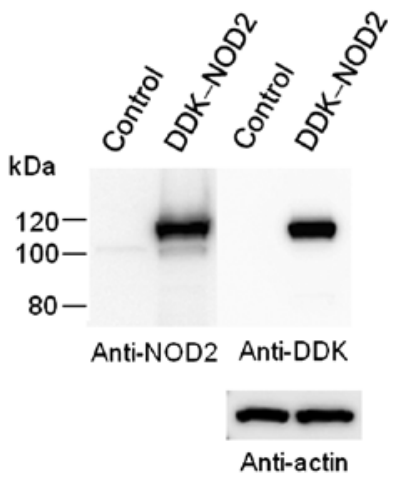

Figure 1. Overexpression of Drosophila headcase (HECA), early B-cell factor 1 (EBF1), or nucleotide-binding oligomerization domain containing 2 (NOD2) in HEK293 cells. HEK293 cells were transfected with expression vectors for DDK epitope-tagged human (A) HECA, (B) EBF1, or (C) NOD2 or with the empty vector alone (control) for $48 \mathrm{~h}$, after which cell lysates were subjected to immunoblot analysis with antibodies to the corresponding proteins, to DDK, and to $\beta$-actin (loading control). Data are representative of eight (HECA, EBF1 and NOD2) or 12 (control) experiments.

sites in 22 genes (with three genes being present in both groups) in atheromatous plaque compared with matched plaque-free intima (Table I). We examined the potential relation of these 33 genes to atherosclerosis, cardiovascular disease, coronary heart disease, or vascular inflammation by searching the PubMed (NCBI) database. Three of the hypomethylated genes (HECA, EBF1 and NOD2) and three of the hypermethylated genes (MAP4K4, ZEBI and $F Y N$ ) have previously been implicated in atherosclerosis or cardiovascular disease (10-15).

Effects of the overexpression of hypomethylated genes or of the attenuated expression of hypermethylated genes on genomewide gene expression. Hypomethylation or hypermethylation of $\mathrm{CpG}$ sites is associated with the up- or downregulation of gene transcription, respectively (3). We therefore examined the effects of overexpression of $H E C A, E B F 1$ or $N O D 2$ or of the shRNA-mediated suppression of $M A P 4 K 4, Z E B 1$ or $F Y N$ expression on genome-wide gene expression in cultured HEK293 cells with the use of a gene expression microarray.

Immunoblot analysis revealed that the abundance of HECA, EBF1 or NOD2 was markedly increased following the transfection of the HEK293 cells with an expression vector for the corresponding human protein (Fig. 1). The overexpression of HECA, EBF1, or NOD2 resulted in a significant $\left(\mathrm{P}<4.80 \times 10^{-7}\right)$ change in the abundance of the 769,980 or 118 transcripts, respectively. We examined these transcripts with the UniGene database (NCBI) and selected 50 corresponding validated or putative protein-coding genes with the lowest P-values for each overexpressed protein (Table II).

The overexpression of HECA significantly altered the expression of genes, including those related to cell proliferation and differentiation [cell division cycle $25 \mathrm{C}(C D C 25 C)$, cyclin $\mathrm{C}$ $(C C N C)$ and $\mathrm{p} 21$ protein $(\mathrm{Cdc} 42 / \mathrm{Rac})$-activated kinase 1 $(P A K 1)$ ], adenosine 3',5'-monophosphate (cAMP) signaling [CREB binding protein $(C R E B B P)], \mathrm{Na}^{+}$and $\mathrm{K}^{+}$transport [ATPase, $\mathrm{Na}^{+} / \mathrm{K}^{+}$transporting, alpha 3 polypeptide $(A T P 1 A 3)$ ], and cyclin-dependent kinase inhibitor $2 \mathrm{~A}(C D K N 2 A)$ regulation [CDKN2A interacting protein $(C D K N 2 A I P)]$. The overexpression of EBF1 significantly affected the expression of genes, including those related to lipid metabolism [phospholipase A2, group IVC (PLA2G4C) and ATP citrate lyase (ACLY)], cell cycle regulation [forkhead box Q1 (FOXQ1), cyclin E1 (CCNE1) and $P A K 1$ ], cell adhesion [cadherin 1, type 1, $(C D H 1)$ ], and inflammatory response CCAAT/enhancer binding protein $(\mathrm{C} / \mathrm{EBP})$, delta $(C E B P D)$. The overexpression of NOD2 significantly altered the expression of genes, including those related to inflammatory response [interleukin-8 $(I L-8)$ ], cell-cell or cell-matrix interaction [lectin, galactoside-binding, soluble, 1 (LGALS1)], cell division and growth [CCNE1 and glypican 3 $(G P C 3)$ ], and $\mathrm{Ca}^{2+}$-channel regulation [calcium channel, voltage-dependent, alpha $2 /$ delta subunit 3 (CACNA2D3)] (Table II).

The abundance of MAP4K4, ZEB1 or FYN was markedly reduced following transfection of the HEK293 cells with an expression vector for a corresponding shRNA (Fig. 2). The depletion of MAP4K4, ZEB1 or FYN resulted in significant $\left(\mathrm{P}<4.80 \times 10^{-7}\right)$ changes in the abundance of the 1485,404 or 411 transcripts, respectively. We examined these transcripts with the NCBI database and selected 50 corresponding validated or putative protein-coding genes with the lowest P-values for each depleted protein (Table III).

The depletion of MAP4K4 markedly altered the expression of genes, including those related to chemokines [chemokine (C-C motif) ligand 5 (CCL5) and chemokine (C-X-C motif) ligand 10 (CXCL1O)], interferons [interferon regulatory factor 7 (IRF7), interferon, lambda 1 (IFNL1) and interferon, beta 1, fibroblast $(I F N B 1)$ ], signal transduction [TGF-beta activated kinase $1 / \mathrm{MAP} 3 \mathrm{~K} 7$ binding protein 2 (TAB2), NF-kappa B activating protein $(N K A P)$ and ras homolog family member $\mathrm{Q}(R H O Q)$ ], leukocyte adhesion [activated leukocyte cell adhesion molecule $(A L C A M)]$, tumor necrosis factor (TNF) release from endothelial cells [nucleobindin 2 $(N U C B 2)]$, and transforming growth factor- $\beta 1$ (TGF- $\beta 1$ ) activity [transforming growth factor, beta receptor associated protein 1 (TGFBRAP1)]. The depletion of ZEB1 significantly affected the expression of genes, including those related to cell cycle regulation [cyclin Y $(C C N Y)$ and cyclin I (CCNI)], TGF- $\beta 1$ activity (TGFBRAPl), TNF release from endothelial cells (NUCB2), cell adhesion [integrin, alpha $\mathrm{V}(I T G A V)$ ], and $\mathrm{K}^{+}$-channel regulation [potassium large conductance calciumactivated channel, subfamily $\mathrm{M}$, beta member $4(K C N M B 4)]$. The depletion of FYN significantly altered the expression of 
Table II. Effects of the overexpression of HECA, EBF1 or NOD2 on gene expression in HEK293 cells.

\begin{tabular}{|c|c|c|c|c|c|c|c|c|}
\hline \multicolumn{3}{|c|}{ HECA } & \multicolumn{3}{|c|}{ EBF1 } & \multicolumn{3}{|c|}{ NOD2 } \\
\hline Gene & $\begin{array}{l}\text { Overexpression/ } \\
\text { control ratio (mean) }\end{array}$ & P-value & Gene & $\begin{array}{l}\text { Overexpression/ } \\
\text { control ratio (mean) }\end{array}$ & P-value & Gene & $\begin{array}{l}\text { Overexpression/ } \\
\text { control ratio (mean) }\end{array}$ & P-value \\
\hline SPIRE2 & 6.13 & $1.0 \times 10^{-16}$ & KIAA1199 & 1.96 & $1.9 \times 10^{-14}$ & HSPA6 & 8.01 & $<1.0 \times 10^{-20}$ \\
\hline REEP2 & 0.64 & $2.1 \times 10^{-15}$ & SPIRE2 & 2.77 & $2.4 \times 10^{-14}$ & HSPA7 & 7.63 & $<1.0 \times 10^{-20}$ \\
\hline C12orf65 & 0.55 & $3.4 \times 10^{-15}$ & STK19 & 0.68 & $4.6 \times 10^{-13}$ & $I L 8$ & 2.75 & $<1.0 \times 10^{-20}$ \\
\hline DMRT3 & 0.69 & $6.7 \times 10^{-13}$ & C12orf65 & 0.70 & $1.5 \times 10^{-12}$ & SPIRE2 & 6.00 & $<1.0 \times 10^{-20}$ \\
\hline$P I G B$ & 1.44 & $8.2 \times 10^{-12}$ & SLC35B3 & 1.35 & $1.1 \times 10^{-11}$ & C12orf65 & 0.48 & $<1.0 \times 10^{-20}$ \\
\hline$I M P 4$ & 0.63 & $3.0 \times 10^{-11}$ & HIST1H4H & 1.47 & $1.3 \times 10^{-11}$ & DNAJB1 & 2.58 & $2.0 \times 10^{-15}$ \\
\hline MAPILC $3 B$ & 1.36 & $4.3 \times 10^{-11}$ & $P G A M 1$ & 1.39 & $3.0 \times 10^{-11}$ & DMRT3 & 0.68 & $2.3 \times 10^{-13}$ \\
\hline NDUFV2 & 0.63 & $5.1 \times 10^{-11}$ & CCNYL1 & 1.27 & $3.9 \times 10^{-11}$ & $R E E P 2$ & 0.69 & $8.4 \times 10^{-13}$ \\
\hline HSPA6 & 1.32 & $5.3 \times 10^{-11}$ & DMRT3 & 0.75 & $4.0 \times 10^{-11}$ & $B A G 3$ & 1.58 & $9.5 \times 10^{-13}$ \\
\hline HSPAIL & 1.32 & $6.6 \times 10^{-11}$ & $P L A 2 G 4 C$ & 0.67 & $6.4 \times 10^{-11}$ & $L G A L S 1$ & 1.33 & $1.8 \times 10^{-11}$ \\
\hline HSPHI & 1.45 & $9.2 \times 10^{-11}$ & BEST1 & 0.71 & $6.7 \times 10^{-11}$ & HSPAIL & 1.61 & $2.0 \times 10^{-11}$ \\
\hline$R P F 2$ & 1.29 & $1.2 \times 10^{-10}$ & HSPAIL & 1.29 & $9.3 \times 10^{-11}$ & HSPHI & 1.91 & $3.8 \times 10^{-11}$ \\
\hline EMILIN2 & 0.74 & $1.4 \times 10^{-10}$ & STOX1 & 1.31 & $1.4 \times 10^{-10}$ & HISTIH4H & 0.69 & $6.1 \times 10^{-11}$ \\
\hline$G A D D 45 G$ & 0.76 & $2.1 \times 10^{-10}$ & FOXQ1 & 1.56 & $2.6 \times 10^{-10}$ & HSPA1B & 1.40 & $8.5 \times 10^{-11}$ \\
\hline$L B R$ & 1.23 & $2.8 \times 10^{-10}$ & RSHL3 & 1.32 & $3.3 \times 10^{-10}$ & ZFAND2A & 1.62 & $9.5 \times 10^{-11}$ \\
\hline RBM4 & 1.21 & $3.6 \times 10^{-10}$ & SNAP25 & 0.70 & $3.6 \times 10^{-10}$ & TTC25 & 0.76 & $2.5 \times 10^{-10}$ \\
\hline CKMT1B & 0.75 & $4.6 \times 10^{-10}$ & $A C L Y$ & 1.18 & $3.6 \times 10^{-10}$ & PSMD8 & 0.59 & $3.4 \times 10^{-10}$ \\
\hline ORC $3 L$ & 1.44 & $6.3 \times 10^{-10}$ & $T S C 22 D 3$ & 1.25 & $3.7 \times 10^{-10}$ & METTL3 & 0.48 & $7.0 \times 10^{-10}$ \\
\hline RAPIGDSI & 1.21 & $6.3 \times 10^{-10}$ & C17orf97 & 0.79 & $4.4 \times 10^{-10}$ & $D E D D 2$ & 1.61 & $8.5 \times 10^{-10}$ \\
\hline BRAT1 & 0.71 & $8.1 \times 10^{-10}$ & $L B R$ & 1.20 & $4.9 \times 10^{-10}$ & C17orf97 & 0.82 & $9.4 \times 10^{-10}$ \\
\hline$R D X$ & 1.41 & $9.4 \times 10^{-10}$ & ZNF823 & 1.36 & $5.2 \times 10^{-10}$ & KIAA0100 & 0.72 & $1.9 \times 10^{-9}$ \\
\hline$C D C 25 C$ & 1.25 & $1.1 \times 10^{-9}$ & $R D X$ & 1.45 & $5.3 \times 10^{-10}$ & BEST1 & 0.80 & $2.1 \times 10^{-9}$ \\
\hline WDR43 & 1.43 & $1.2 \times 10^{-9}$ & FAM53C & 0.81 & $5.7 \times 10^{-10}$ & $H I S T 2 H 4 B$ & 0.73 & $2.1 \times 10^{-9}$ \\
\hline HNRNPCL1 & 0.84 & $1.6 \times 10^{-9}$ & $F A M 3 C$ & 1.21 & $5.9 \times 10^{-10}$ & CHORDC1 & 1.38 & $2.3 \times 10^{-9}$ \\
\hline ZNF184 & 1.39 & $1.7 \times 10^{-9}$ & $\mathrm{CDH}$ & 1.27 & $5.9 \times 10^{-10}$ & $D Y N L R B 2$ & 0.81 & $2.4 \times 10^{-9}$ \\
\hline CCNC & 1.32 & $1.9 \times 10^{-9}$ & ACTAl & 0.73 & $6.6 \times 10^{-10}$ & SNAP25 & 0.75 & $3.4 \times 10^{-9}$ \\
\hline SCAND3 & 1.35 & $2.0 \times 10^{-9}$ & $C E B P D$ & 1.32 & $7.4 \times 10^{-10}$ & SPATA2L & 0.77 & $5.4 \times 10^{-9}$ \\
\hline$B R D 8$ & 1.22 & $2.0 \times 10^{-9}$ & $A N K M Y 2$ & 1.41 & $8.8 \times 10^{-10}$ & ARHGEF37 & 0.78 & $5.9 \times 10^{-9}$ \\
\hline DOCK10 & 0.79 & $2.1 \times 10^{-9}$ & REEP2 & 0.80 & $1.0 \times 10^{-9}$ & SLC25A42 & 0.83 & $6.9 \times 10^{-9}$ \\
\hline$C R E B B P$ & 1.24 & $2.1 \times 10^{-9}$ & $G A D D 45 G$ & 0.76 & $1.1 \times 10^{-9}$ & INSM2 & 0.76 & $7.5 \times 10^{-9}$ \\
\hline$U G T 2 B 11$ & 0.83 & $2.6 \times 10^{-9}$ & COL8A2 & 0.80 & $1.4 \times 10^{-9}$ & IFIT2 & 1.35 & $7.7 \times 10^{-9}$ \\
\hline ATP1A3 & 0.80 & $3.6 \times 10^{-9}$ & SIGMAR1 & 1.20 & $1.6 \times 10^{-9}$ & HIST2H4A & 0.61 & $1.0 \times 10^{-8}$ \\
\hline RHBDD2 & 1.16 & $4.4 \times 10^{-9}$ & $B R D 8$ & 1.24 & $1.8 \times 10^{-9}$ & C16orf93 & 0.79 & $1.1 \times 10^{-8}$ \\
\hline GFM2 & 1.32 & $4.5 \times 10^{-9}$ & $G A B B R 2$ & 1.41 & $1.8 \times 10^{-9}$ & $O V G P 1$ & 0.75 & $1.3 \times 10^{-8}$ \\
\hline FOXI3 & 0.84 & $5.0 \times 10^{-9}$ & GAPDH & 1.19 & $1.8 \times 10^{-9}$ & $P I G B$ & 1.36 & $1.3 \times 10^{-8}$ \\
\hline ZNF823 & 1.23 & $5.2 \times 10^{-9}$ & $P C G F 3$ & 0.78 & $2.0 \times 10^{-9}$ & $R E L B$ & 1.30 & $1.7 \times 10^{-8}$ \\
\hline SENP6 & 1.40 & $5.3 \times 10^{-9}$ & CCNE1 & 1.18 & $2.2 \times 10^{-9}$ & THOC3 & 1.23 & $1.9 \times 10^{-8}$ \\
\hline SRFBP1 & 1.24 & $5.6 \times 10^{-9}$ & PREPL & 1.28 & $2.2 \times 10^{-9}$ & NDUFV2 & 0.69 & $2.0 \times 10^{-8}$ \\
\hline THOC3 & 1.24 & $5.6 \times 10^{-9}$ & BMP6 & 1.43 & $2.2 \times 10^{-9}$ & $H S P A 4 L$ & 1.39 & $2.0 \times 10^{-8}$ \\
\hline$L Y 6 H$ & 0.83 & $5.6 \times 10^{-9}$ & ENO3 & 0.82 & $2.3 \times 10^{-9}$ & RETNLB & 0.76 & $2.2 \times 10^{-8}$ \\
\hline PAK1 & 1.26 & $5.7 \times 10^{-9}$ & $M M P 23 B$ & 0.82 & $2.3 \times 10^{-9}$ & $U G T 2 B 11$ & 0.86 & $2.6 \times 10^{-8}$ \\
\hline$N R A S$ & 1.27 & $5.7 \times 10^{-9}$ & $V G F$ & 0.81 & $2.5 \times 10^{-9}$ & DOCK10 & 0.80 & $3.4 \times 10^{-8}$ \\
\hline$A N K R D 7$ & 0.86 & $5.9 \times 10^{-9}$ & REM2 & 0.77 & $2.6 \times 10^{-9}$ & CCNE1 & 1.12 & $3.4 \times 10^{-8}$ \\
\hline C20orf 27 & 0.82 & $6.0 \times 10^{-9}$ & SCAND3 & 1.37 & $2.8 \times 10^{-9}$ & $G P C 3$ & 1.24 & $3.8 \times 10^{-8}$ \\
\hline$C D K N 2 A I P$ & 1.3 & $7.3 \times 10^{-9}$ & $P A K 1$ & 1.40 & $3.1 \times 10^{-9}$ & $I Q C K$ & 0.82 & $4.8 \times 10^{-8}$ \\
\hline$D L K 2$ & 0.87 & $7.7 \times 10^{-9}$ & $Z P 3$ & 0.84 & $3.3 \times 10^{-9}$ & C9orf116 & 0.86 & $5.1 \times 10^{-8}$ \\
\hline TCEAl & 1.25 & $8.2 \times 10^{-9}$ & TPD52L2 & 0.83 & $3.4 \times 10^{-9}$ & $Z P 3$ & 0.85 & $5.3 \times 10^{-8}$ \\
\hline C20orf194 & 0.86 & $8.3 \times 10^{-9}$ & CCDC155 & 0.81 & $3.6 \times 10^{-9}$ & CACNA2D3 & 0.75 & $6.1 \times 10^{-8}$ \\
\hline PSMD4 & 1.21 & $8.3 \times 10^{-9}$ & $M A P 1 L C 3 B$ & 1.29 & $3.7 \times 10^{-9}$ & DHRS2 & 0.83 & $6.5 \times 10^{-8}$ \\
\hline PCDHA@ & 0.84 & $9.1 \times 10^{-9}$ & PSMA1 & 1.18 & $3.8 \times 10^{-9}$ & $P G A M 1$ & 1.32 & $7.5 \times 10^{-8}$ \\
\hline
\end{tabular}

Microarray-based genome-wide analysis of gene expression was performed for HEK293 cells transfected as in Fig. 1. Data were obtained from eight (HECA, EBF1, NOD2) or 12 (control) experiments. Transcripts whose abundance was significantly $\left(\mathrm{P}<4.80 \times 10^{-7}\right)$ changed by overexpression of HECA, EBF1 or NOD2 were examined with the UniGene database (NCBI), and 50 corresponding validated or putative protein-coding genes with the lowest P-values for each overexpressed protein are listed. 

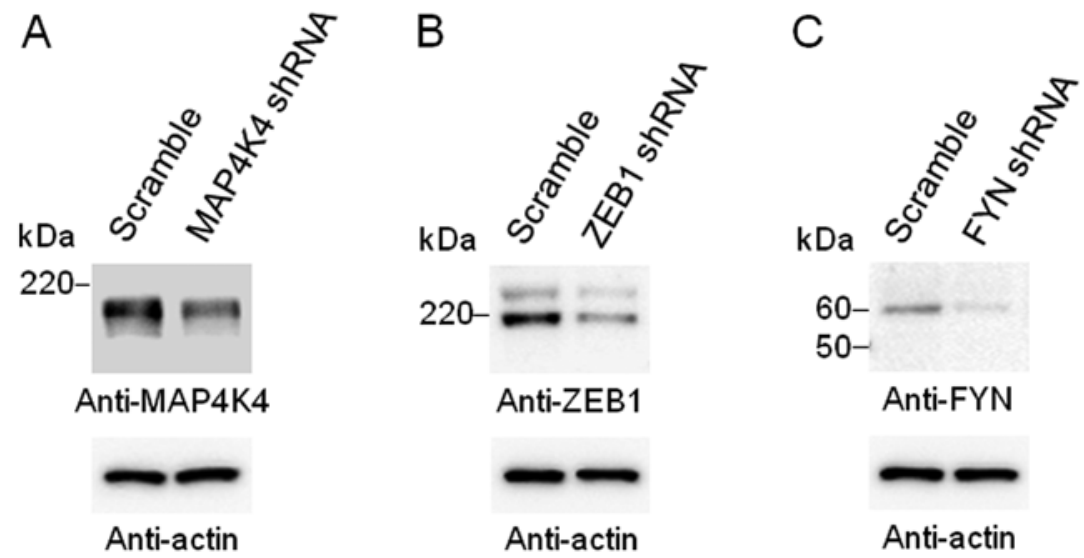

Figure 2. Depletion of human mitogen-activated protein kinase kinase kinase kinase 4 (MAP4K4), zinc finger E-box binding homeobox 1 (ZEB1), or FYN in HEK 293 cells. HEK293 cells were transfected with vectors for human (A) $M A P 4 K 4$, (B) ZEB1, or (C) $F Y N$ short hairpin RNAs (shRNAs) or for a scrambled shRNA for $72 \mathrm{~h}$, after which cell lysates were subjected to immunoblot analysis with antibodies to the corresponding proteins or to $\beta$-actin (loading control). Data are representative of eight $(M A P 4 K 4, Z E B 1$ and $F Y N)$ or 12 (control) experiments.

genes, including those related to cell cycle regulation $[C C N Y$ and cyclin-dependent kinase 11B $(C D K 11 B)]$, signal transduction [TAB2, RHOQ, NKAP, dual specificity phosphatase 8 (DUSP8) and CDP-diacylglycerol synthase (phosphatidate cytidylyltransferase) 1 (CDS1)], energy metabolism [stearoylCoA desaturase 5 (SCD5)], TGF- $\beta 1$ activity (TGFBRAP1), and peroxisome proliferator-activated receptor $\gamma$ (PPAR $\gamma)$ activity [peroxisome proliferator-activated receptor gamma, coactivator 1 beta $(P P A R G C 1 B)]$ (Table III).

\section{Discussion}

In this study, we demonstrate that $H E C A, E B F 1$ and NOD2 are significantly hypomethylated, whereas $M A P 4 K 4, Z E B 1$ and $F Y N$ are significantly hypermethylated, in genomic DNA isolated from atheromatous plaque compared with that from matched plaque-free intima. We demonstrate that the overexpression of HECA, EBF1 or NOD2 or the depletion of MAP4K4, ZEB1 or FYN in cultured HEK293 cells results in significant changes in the expression of various atherosclerosis-related genes.

HECA. Evidence has suggested that HECA plays an important role in human carcinogenesis (16). HECA has also previously been found to be related to coronary heart disease, with HECA expression being increased in the atherosclerotic aortic wall (10). In this study, we demonstrated that HECA was significantly hypomethylated in atheromatous plaque and that the overexpression of HECA in HEK293 cells resulted in the increased expression of genes related to cell proliferation $(C D C 25 C, C C N C$ and $P A K 1)$, a process important in the development of atherosclerosis in the arterial intima. The overexpression of HECA also increased the expression of a gene related to cAMP signaling $(C R E B B P)$, which may play an important role in the pathogenesis of atherosclerosis (17). In addition, the overexpression of HECA increased the expression of a gene related to $\mathrm{CDKN} 2 \mathrm{~A}$ regulation $(C D K N 2 A I P)$. Given that $C D K N 2 A$ is a susceptibility locus for coronary heart disease and myocardial infarction (18), the increased expression of CDKN2AIP may be related to these conditions.
$E B F 1$. EBF1 is an important determinant of early B lymphopoiesis and as such contributes to hematopoiesis and immunity (19). Analysis of knockout mice has also identified a role for EBF1 in lipid metabolism and phenotypes related to cardiovascular disease. EBF1-deficient mice manifest lipodystrophy characterized by a marked decrease in the amount of white adipose tissue, as well as an increase in yellow adipose tissue in bone marrow compared with wild-type controls (20), consistent with the notion that EBF1 participates in terminal adipocyte differentiation and the initiation of adipocyte development (21). The expression of EBF1 has previously been found to be increased in visceral fat and the atherosclerotic aortic wall (10), and polymorphisms of EBF1 have been shown to be related both to the plasma concentration of low density lipoprotein-cholesterol and to coronary atherosclerosis (11). In this study, we demonstrated that $E B F 1$ was significantly hypomethylated in atheromatous plaque and that the overexpression of EBF1 in HEK293 cells resulted in the increased expression of genes related to cell proliferation (FOXQ1, CCNE1 and $P A K 1)$, to inflammatory response $(C E B P D)$, and to cell adhesion $(C D H 1)$.

NOD2. NOD1 and NOD2 regulate the activation of nuclear factor of $\kappa$ light polypeptide gene enhancer in $B$ cells 1 (NFKB1) in human fibroblast and aortic endothelial cell lines in response to infection with Chlamydophila pneumoniae, one of the most common bacterial species detected in atherosclerotic plaques (22). NOD2 senses bacterial molecules and activates NFKB1-dependent gene expression through the RIP2-IKK pathway $(23,24)$. The NFKB1 pathway contributes to the upregulation of the expression of pro-inflammatory molecules, resulting in amplification of inflammation and stimulation of both adaptive and innate immune responses (24). NOD2 may thus be a key regulator of vascular inflammation and the development of atherosclerosis (25). Genetic variants of NOD2 have been related to coronary heart disease (12). In this study, we showed that NOD2 was significantly hypomethylated in atheromatous plaque and that the overexpression of NOD2 in HEK293 cells resulted in the increased expression of genes related to inflammatory response $(I L-8)$, to cell-cell or cell- 
Table III. Effects of the depletion of MAP4K4, ZEB1 or FYN on gene expression in HEK293 cells.

\begin{tabular}{|c|c|c|c|c|c|c|c|c|}
\hline \multicolumn{3}{|c|}{ MAP4K4 } & \multicolumn{3}{|c|}{ ZEB1 } & \multicolumn{3}{|c|}{ FYN } \\
\hline Gene & $\begin{array}{l}\text { Depletion/control } \\
\text { ratio (mean) }\end{array}$ & P-value & Gene & $\begin{array}{l}\text { Depletion/control } \\
\text { ratio (mean) }\end{array}$ & P-value & Gene & $\begin{array}{l}\text { Depletion/control } \\
\text { ratio (mean) }\end{array}$ & P-value \\
\hline DDX60 & 2.08 & $<1.0 \times 10^{-20}$ & LAPTM4A & 1.39 & $1.2 \times 10^{-11}$ & $C C N Y$ & 0.62 & $3.3 \times 10^{-14}$ \\
\hline$C C L 5$ & 3.58 & $<1.0 \times 10^{-20}$ & ZNF638 & 1.46 & $3.2 \times 10^{-11}$ & $D C B L D 1$ & 0.56 & $3.4 \times 10^{-13}$ \\
\hline$I F I H 1$ & 2.75 & $<1.0 \times 10^{-20}$ & CCNY & 0.69 & $9.6 \times 10^{-11}$ & FOXO3 & 0.62 & $4.8 \times 10^{-12}$ \\
\hline$I S G 15$ & 2.76 & $<1.0 \times 10^{-20}$ & SPIRE2 & 1.89 & $1.2 \times 10^{-10}$ & SPIRE2 & 4.45 & $3.0 \times 10^{-11}$ \\
\hline$B S T 2$ & 2.06 & $3.3 \times 10^{-16}$ & TNPO3 & 0.71 & $1.6 \times 10^{-10}$ & GNA11 & 0.53 & $3.4 \times 10^{-11}$ \\
\hline EPSTI1 & 2.13 & $1.0 \times 10^{-15}$ & ELOVL7 & 1.44 & $3.8 \times 10^{-10}$ & $T A B 2$ & 1.29 & $3.9 \times 10^{-11}$ \\
\hline SPIRE2 & 3.03 & $1.3 \times 10^{-15}$ & $R F K$ & 1.17 & $4.7 \times 10^{-10}$ & ZNF728 & 2.39 & $4.3 \times 10^{-11}$ \\
\hline IRF7 & 1.84 & $1.7 \times 10^{-15}$ & HNRNPCLI & 1.55 & $7.2 \times 10^{-10}$ & NDUFS1 & 0.71 & $5.0 \times 10^{-11}$ \\
\hline PPP1R15A & 1.77 & $5.2 \times 10^{-15}$ & $M N X 1$ & 1.35 & $7.2 \times 10^{-10}$ & TNPO3 & 0.78 & $5.9 \times 10^{-11}$ \\
\hline DHRS2 & 2.72 & $6.6 \times 10^{-15}$ & SNHG12 & 1.32 & $8.8 \times 10^{-10}$ & $S L C 3 A 2$ & 1.38 & $1.2 \times 10^{-10}$ \\
\hline$I S G 20$ & 1.53 & $9.0 \times 10^{-15}$ & HIVEP2 & 1.37 & $1.4 \times 10^{-9}$ & $V R K 3$ & 0.68 & $1.3 \times 10^{-10}$ \\
\hline HIFO & 1.70 & $1.2 \times 10^{-14}$ & FOXO3 & 0.62 & $1.7 \times 10^{-9}$ & $R H O Q$ & 0.56 & $1.3 \times 10^{-10}$ \\
\hline GORAB & 1.48 & $1.3 \times 10^{-14}$ & MAGEHI & 1.24 & $2.4 \times 10^{-9}$ & ZNF275 & 0.60 & $2.1 \times 10^{-10}$ \\
\hline PIAS1 & 1.98 & $1.6 \times 10^{-14}$ & ZKSCAN1 & 0.63 & $2.7 \times 10^{-9}$ & NKAP & 1.53 & $3.2 \times 10^{-10}$ \\
\hline$W A C$ & 1.55 & $2.5 \times 10^{-14}$ & SIPA1L1 & 1.38 & $3.1 \times 10^{-9}$ & PLEKHB 2 & 0.73 & $3.4 \times 10^{-10}$ \\
\hline IFIT2 & 4.34 & $5.0 \times 10^{-14}$ & LYRM2 & 0.67 & $3.2 \times 10^{-9}$ & $S P O P$ & 0.60 & $3.7 \times 10^{-10}$ \\
\hline IFI6 & 2.97 & $5.1 \times 10^{-14}$ & CCNI & 0.73 & $3.6 \times 10^{-9}$ & LYRM2 & 0.68 & $3.9 \times 10^{-10}$ \\
\hline IFNLI & 2.11 & $6.7 \times 10^{-14}$ & LMO4 & 1.30 & $3.6 \times 10^{-9}$ & FICD & 0.71 & $4.5 \times 10^{-10}$ \\
\hline HERC6 & 1.46 & $7.2 \times 10^{-14}$ & CSTF2 & 1.22 & $3.7 \times 10^{-9}$ & FAM161A & 0.55 & $5.8 \times 10^{-10}$ \\
\hline HERC5 & 1.65 & $7.4 \times 10^{-14}$ & GPR101 & 1.32 & $3.9 \times 10^{-9}$ & $I G D C C 3$ & 0.74 & $6.2 \times 10^{-10}$ \\
\hline IFIT1 & 2.75 & $8.8 \times 10^{-14}$ & HIST1H1C & 1.36 & $3.9 \times 10^{-9}$ & $C D K 11 B$ & 0.67 & $7.5 \times 10^{-10}$ \\
\hline IFIT3 & 1.45 & $9.2 \times 10^{-14}$ & LYPD1 & 1.25 & $4.4 \times 10^{-9}$ & ZNF681 & 1.62 & $7.6 \times 10^{-10}$ \\
\hline DDX58 & 1.48 & $1.0 \times 10^{-13}$ & FICD & 0.75 & $4.7 \times 10^{-9}$ & NDUFB6 & 0.72 & $8.4 \times 10^{-10}$ \\
\hline IFITM3 & 2.36 & $1.1 \times 10^{-13}$ & $A N P 32 A$ & 1.44 & $5.1 \times 10^{-9}$ & SLIT2 & 0.58 & $8.4 \times 10^{-10}$ \\
\hline$T A B 2$ & 1.36 & $1.7 \times 10^{-13}$ & $T E A D 4$ & 0.87 & $5.2 \times 10^{-9}$ & TECPRI & 0.67 & $9.4 \times 10^{-10}$ \\
\hline BEST1 & 1.76 & $2.2 \times 10^{-13}$ & $M 6 P R$ & 0.72 & $5.3 \times 10^{-9}$ & PCYOX1 & 0.64 & $1.0 \times 10^{-9}$ \\
\hline$A L C A M$ & 1.43 & $2.3 \times 10^{-13}$ & HOXC6 & 1.35 & $5.3 \times 10^{-9}$ & DUSP8 & 0.63 & $1.1 \times 10^{-9}$ \\
\hline LMO4 & 1.39 & $4.8 \times 10^{-13}$ & $B C A S 2$ & 1.40 & $5.6 \times 10^{-9}$ & ZNF787 & 1.29 & $1.1 \times 10^{-9}$ \\
\hline ZNF787 & 1.32 & $6.0 \times 10^{-13}$ & TGFBRAPI & 0.69 & $5.9 \times 10^{-9}$ & SCD5 & 0.76 & $1.2 \times 10^{-9}$ \\
\hline IFITM1 & 1.74 & $6.0 \times 10^{-13}$ & AKIRIN1 & 0.77 & $5.9 \times 10^{-9}$ & $A U P 1$ & 1.39 & $1.4 \times 10^{-9}$ \\
\hline LAPTM4A & 1.46 & $6.3 \times 10^{-13}$ & PLEKHB2 & 0.74 & $6.1 \times 10^{-9}$ & HIVEP2 & 1.42 & $1.5 \times 10^{-9}$ \\
\hline IFI44L & 1.93 & $9.2 \times 10^{-13}$ & $N U C B 2$ & 1.41 & $6.4 \times 10^{-9}$ & $F Y N$ & 0.73 & $1.5 \times 10^{-9}$ \\
\hline ZNF274 & 1.24 & $1.0 \times 10^{-12}$ & RPS26 & 1.24 & $6.6 \times 10^{-9}$ & AKIRIN1 & 0.78 & $1.7 \times 10^{-9}$ \\
\hline$O V G P 1$ & 1.62 & $1.3 \times 10^{-12}$ & ITGAV & 1.32 & $6.6 \times 10^{-9}$ & TGFBRAP1 & 0.66 & $2.4 \times 10^{-9}$ \\
\hline OASL & 1.60 & $1.4 \times 10^{-12}$ & PRPF40A & 0.65 & $7.5 \times 10^{-9}$ & $H I P K 2$ & 0.64 & $2.6 \times 10^{-9}$ \\
\hline$N K A P$ & 1.57 & $1.4 \times 10^{-12}$ & HOOK1 & 0.63 & $7.5 \times 10^{-9}$ & LAPTM4A & 1.48 & $3.1 \times 10^{-9}$ \\
\hline CXCL10 & 2.06 & $1.6 \times 10^{-12}$ & $S Y K$ & 0.79 & $8.1 \times 10^{-9}$ & $P D P R$ & 0.75 & $4.2 \times 10^{-9}$ \\
\hline$B C A S 2$ & 1.57 & $1.7 \times 10^{-12}$ & ATP6V1G1 & 1.49 & $8.2 \times 10^{-9}$ & $C D S 1$ & 0.67 & $4.2 \times 10^{-9}$ \\
\hline IFITM2 & 1.55 & $2.0 \times 10^{-12}$ & $G N L 3 L$ & 0.64 & $9.4 \times 10^{-9}$ & PRPF40A & 0.74 & $4.7 \times 10^{-9}$ \\
\hline PLEKHB2 & 0.70 & $2.2 \times 10^{-12}$ & PCDH17 & 0.79 & $9.6 \times 10^{-9}$ & $D A B 2 I P$ & 0.74 & $4.8 \times 10^{-9}$ \\
\hline TAOK1 & 0.55 & $2.2 \times 10^{-12}$ & $N A B 1$ & 1.32 & $9.7 \times 10^{-9}$ & $G N L 3 L$ & 0.65 & $5.2 \times 10^{-9}$ \\
\hline IFNBI & 3.43 & $2.4 \times 10^{-12}$ & $R P L 7$ & 1.49 & $9.9 \times 10^{-9}$ & PPARGC1B & 0.80 & $5.3 \times 10^{-9}$ \\
\hline RHOQ & 0.53 & $2.8 \times 10^{-12}$ & $H M G N 3$ & 1.29 & $1.0 \times 10^{-8}$ & SNRPD3 & 0.64 & $5.3 \times 10^{-9}$ \\
\hline$R A D 23 A$ & 1.42 & $4.4 \times 10^{-12}$ & MED9 & 1.32 & $1.1 \times 10^{-8}$ & $N F X L 1$ & 1.33 & $5.3 \times 10^{-9}$ \\
\hline$T A F 1 D$ & 1.77 & $5.2 \times 10^{-12}$ & GMCL1 & 0.66 & $1.1 \times 10^{-8}$ & UTRN & 1.31 & $5.6 \times 10^{-9}$ \\
\hline RIOK3 & 1.36 & $5.4 \times 10^{-12}$ & $V R K 3$ & 0.72 & $1.1 \times 10^{-8}$ & ZNF326 & 0.65 & $5.6 \times 10^{-9}$ \\
\hline$N U C B 2$ & 1.53 & $7.3 \times 10^{-12}$ & KCNMB4 & 1.31 & $1.2 \times 10^{-8}$ & $R A B 9 B$ & 0.76 & $6.0 \times 10^{-9}$ \\
\hline TGFBRAP1 & 0.63 & $7.4 \times 10^{-12}$ & $E I F 2 A K 2$ & 0.65 & $1.2 \times 10^{-8}$ & $D Z I P 1$ & 0.70 & $6.0 \times 10^{-9}$ \\
\hline INTS8 & 1.39 & $8.9 \times 10^{-12}$ & $M E A 1$ & 1.23 & $1.3 \times 10^{-8}$ & $D L S T$ & 0.69 & $6.1 \times 10^{-9}$ \\
\hline HNRNPCL1 & 1.79 & $1.1 \times 10^{-11}$ & $R A B 3 B$ & 0.82 & $1.6 \times 10^{-8}$ & TSEN15 & 1.22 & $6.5 \times 10^{-9}$ \\
\hline
\end{tabular}

Microarray-based genome-wide analysis of gene expression was performed for HEK293 cells transfected as in Fig. 2. Data were obtained from eight (MAP4K4, ZEB1, FYN) or 12 (control) experiments. Transcripts whose abundance was significantly $\left(\mathrm{P}<4.80 \times 10^{-7}\right)$ changed by depletion of MAP4K4, ZEB1 or FYN were examined with the UniGene database (NCBI), and 50 corresponding validated or putative protein-coding genes with the lowest P-values are listed. 
matrix interaction ( $L G A L S 1)$, and to cell division and growth (CCNE1 and GPC3).

MAP4K4. MAP4K4 is a member of the serine-threonine protein kinase family and specifically activates signaling by MAPK8 (also known as JNK1). MAP4K4 may function through the MAP3K7-MAP2K4-MAP2K7 kinase cascade and mediates signaling triggered by TNF (NCBI). A polymorphism of $M A P 4 K 4$ has been related to carotid artery intima-media thickness for women receiving hormone replacement therapy (13). In this study, we demonstrated that MAP4K4 was significantly hypermethylated in atheromatous plaque and that the shRNAmediated depletion of MAP4K4 in HEK293 cells resulted both in the increased expression of genes related to chemokines (CCL5 and CXCL10), interferons (IRF7, IFNL1 and IFNB1), leukocyte adhesion ( $A L C A M)$, TNF release from endothelial cells (NUCB2), and the activation of NFKB1 signaling (TAB2 and $N K A P$ ), all of which are also related to vascular inflammation, as well as in the attenuation of the expression of a gene related to TGF- $\beta 1$ activity (TGFBRAPI).

ZEB1. ZEB1 is a zinc finger transcription factor expressed in various cell types, including vascular smooth muscle cells (26). Silencing of $Z E B 1$ by RNA interference increased expression of inflammatory genes such as that for prostaglandin-endoperoxide synthase 2 in vascular smooth muscle cells (14), suggesting that ZEB1 negatively regulates the expression of such genes. In this study, we showed that ZEBI was significantly hypermethylated in atheromatous plaque and that the depletion of ZEB1 in HEK293 cells resulted both in the increased expression of genes related to TNF release from endothelial cells (NUCB2) and cell adhesion (ITGAV), as well as in the reduced expression of a gene related to TGF- $\beta 1$ activity (TGFBRAPI).

FYN oncogene related to SRC, FGR, YES (FYN). FYN is a member of a family of protein tyrosine kinases that also serve as oncoproteins. It is a membrane-associated protein and has been implicated in the control of cell proliferation. It associates with the p85 subunit of phosphatidylinositol 3-kinase and with FYN binding protein (NCBI database), and has been shown to play a role in the activation of platelets (15). The expression of $F Y N$ has previously been found to be decreased in the atherosclerotic aortic wall (10). In this study, we demonstrated that FYN was significantly hypermethylated in atheromatous plaque and that the depletion of FYN in HEK293 cells resulted in the increased expression of genes related to NFKB1 signaling (TAB2 and $N K A P)$ and in the reduced expression of genes related to TGF- $\beta 1$ (TGFBRAPI) or PPAR $\gamma(P P A R G C 1 B)$ activity.

Although our microarray-based analysis of genome-wide gene expression revealed significant alterations in the expression of diverse genes in response to the overexpression of $H E C A, E B F 1$ or $N O D 2$ or to the suppression of the expression of $M A P 4 K 4, Z E B 1$ or $F Y N$, our results suggest that the up- or downregulation of genes related to vascular inflammation or atherogenesis is prominent among such alterations.

Given that epigenetic modification has been shown to be tissue-specific, heterogeneity in DNA methylation levels has been observed among different cell and tissue types $(27,28)$. The aortic intima comprises heterogeneous cell types. The plaque-free intima thus contains endothelial cells, smooth muscle cells, fibroblasts, and monocytes-macrophages, whereas the intima of atheromatous plaque contains these cell types as well as foam cells. Given that it is difficult to isolate individual cell types, such as foam cells from atheromatous plaque, we analyzed the DNA methylation patterns of genomic DNA samples extracted from atheromatous plaque and plaquefree intima. Given that interindividual variation in DNA methylation has also been detected for the same type of cell from the same type of tissue of unrelated individuals $(28,29)$, we performed intraindividual paired comparisons between atheromatous plaque and corresponding plaque-free tissue to avoid the effects of such variation.

There were several limitations to the present study: i) the aortic intima samples comprised heterogeneous cell types. ii) Given the small sample size of the study, the statistical power of the genome-wide analysis of DNA methylation was not optimal. iii) The molecular mechanisms underlying the effects of DNA methylation identified in the present study on the development of atherosclerosis have not been determined definitively. iv) Validation of our findings will require their replication with other independent subject panels or ethnic groups.

In conclusion, our present results suggest that $H E C A$, $E B F 1$ and NOD2 are significantly hypomethylated, whereas $M A P 4 K 4, Z E B 1$ and $F Y N$ are hypermethylated, in atheromatous plaque lesions compared with plaque-free intima. The overexpression of these hypomethylated genes or the silencing of the hypermethylated genes in cultured cells resulted in significant changes in the expression of various atherosclerosis-related genes. Our findings thus suggest that epigenetic mechanisms may contribute to the pathogenesis of atherosclerosis.

\section{Acknowledgements}

This study was supported by a Grant-in-Aid for Scientific Research from the Ministry of Education, Culture, Sports, Science and Technology of Japan (no. 24590746 to Y.Y.)

\section{References}

1. Tsai PC, Spector TD and Bell JT: Using epigenome-wide association scans of DNA methylation in age-related complex human traits. Epigenomics 4: 511-526, 2012.

2. Rakyan VK, Down TA, Balding DJ and Beck S: Epigenomewide association studies for common human diseases. Nat Rev Genet 12: 529-541, 2011.

3. Handy DE, Castro R and Loscalzo J: Epigenetic modifications: basic mechanisms and role in cardiovascular disease. Circulation 123: 2145-2156, 2011.

4. Turunen MP, Aavik E and Ylä-Herttuala S: Epigenetics and atherosclerosis. Biochim Biophys Acta 1790: 886-891, 2009.

5. Talens RP, Jukema JW, Trompet S, et al: Hypermethylation at loci sensitive to the prenatal environment is associated with increased incidence of myocardial infarction. Int J Epidemiol 41: 106-115, 2012.

6. Castillo-Díaz SA, Garay-Sevilla ME, Hernández-González MA, Solís-Martínez MO and Zaina S: Extensive demethylation of normally hypermethylated $\mathrm{CpG}$ islands occurs in human atherosclerotic arteries. Int J Mol Med 26: 691-700, 2010.

7. Bibikova M, Barnes B, Tsan C, et al: High density DNA methylation array with single CpG site resolution. Genomics 98: 288-295, 2011.

8. Sandoval J, Heyn H, Moran S, et al: Validation of a DNA methylation microarray for $450,000 \mathrm{CpG}$ sites in the human genome. Epigenetics 6: 692-702, 2011. 
9. Durocher Y, Perret S and Kamen A: High-level and highthroughput recombinant protein production by transient transfection of suspension-growing human 293-EBNA1 cells. Nucleic Acids Res 30: E9, 2002.

10. Hägg S, Skogsberg J, Lundström J, et al: Multi-organ expression profiling uncovers a gene module in coronary artery disease involving transendothelial migration of leukocytes and LIM domain binding 2: the Stockholm Atherosclerosis Gene Expression (STAGE) study. PLoS Genet 5: e1000754, 2009.

11. Nolan DK, Sutton B, Haynes C, et al: Fine mapping of a linkage peak with integration of lipid traits identifies novel coronary artery disease genes on chromosome 5. BMC Genet 13: 12, 2012.

12. Galluzzo S, Patti G, Dicuonzo G, et al: Association between NOD2/CARD15 polymorphisms and coronary artery disease: a case-control study. Hum Immunol 72: 636-640, 2011.

13. Miller VM, Petterson TM, Jeavons EN, et al: Genetic polymorphisms associated with carotid artery intima-media thickness and coronary artery calcification in women of the Kronos Early Estrogen Prevention Study. Physiol Genomics 45: 79-88, 2013.

14. Reddy MA, Jin W, Villeneuve L, et al: Pro-inflammatory role of microRNA-200 in vascular smooth muscle cells from diabetic mice. Arterioscler Thromb Vasc Biol 32: 721-729, 2012.

15. Chen K, Li W, Major J, Rahaman SO, Febbraio M and Silverstein RL: Vav guanine nucleotide exchange factors link hyperlipidemia and a prothrombotic state. Blood 117: 5744-5750, 2011.

16. Dowejko A, Bauer RJ, Müller-Richter UD and Reichert TE: The human homolog of the Drosophila headcase protein slows down cell division of head and neck cancer cells. Carcinogenesis 30: 1678-1685, 2009.

17. Fantidis P: The role of intracellular 3'5'-cyclic adenosine monophosphate (cAMP) in atherosclerosis. Curr Vasc Pharmacol 8: 464-472, 2010.

18. CARDIoGRAMplusC4D Consortium, Deloukas P, Kanoni S, Willenborg C, et al: Large-scale association analysis identifies new risk loci for coronary artery disease. Nat Genet 45: 25-33, 2013.
19. Lukin K, Fields S, Hartley J and Hagman J: Early B cell factor: regulator of B lineage specification and commitment. Semin Immunol 20: 221-227, 2008.

20. Fretz JA, Nelson T, Xi Y, Adams DJ, Rosen CJ and Horowitz MC: Altered metabolism and lipodystrophy in the early B-cell factor 1-deficient mouse. Endocrinology 151: 1611-1621, 2010.

21. Akerblad P, Lind U, Liberg D, Bamberg K and Sigvardsson M: Early B-cell factor $(\mathrm{O} / \mathrm{E}-1)$ is a promoter of adipogenesis and involved in control of genes important for terminal adipocyte differentiation. Mol Cell Biol 22: 8015-8025, 2002.

22. Opitz B, Förster S, Hocke AC, et al: Nod1-mediated endothelial cell activation by Chlamydophila pneumoniae. Circ Res 96: 319-326, 2005.

23. Girardin SE, Boneca IG, Viala J, et al: Nod2 is a general sensor of peptidoglycan through muramyl dipeptide (MDP) detection. J Biol Chem 278: 8869-8872, 2003.

24. Inohara N, Koseki T, Lin J, et al: An induced proximity model for NF-kappa B activation in the Nod1/RICK and RIP signaling pathways. J Biol Chem 275: 27823-27831, 2000.

25. Collins T and Cybulsky MI: NF- $\kappa$ B: pivotal mediator or innocent bystander in atherogenesis? J Clin Invest 107: 255-264, 2001.

26. Vandewalle $C$, Van Roy F and Berx G: The role of the ZEB family of transcription factors in development and disease. Cell Mol Life Sci 66: 773-787, 2009.

27. Davies MN, Volta M, Pidsley R, et al: Functional annotation of the human brain methylome identifies tissue-specific epigenetic variation across brain and blood. Genome Biol 13: R43, 2012.

28. Christensen BC, Houseman EA, Marsit CJ, et al: Aging and environmental exposures alter tissue-specific DNA methylation dependent upon CpG island context. PLoS Genet 5: e1000602, 2009.

29. Bell JT, Pai AA, Pickrell JK, et al: DNA methylation patterns associate with genetic and gene expression variation in HapMap cell lines. Genome Biol 12: R10, 2011. 